\title{
REVIEW
}

\section{Medical photography and genitourinary surgery}

F. FERRO and D.A. BLOOM*

'Bambino Gesu' Children's Hospital, Rome, Italy and *University of Michigan, Ann Arbor, Michigan, USA

\section{Introduction}

Physicians have been quick to appreciate the pleasures and applications of photography. In 1863 Oliver Wendell Holmes, one of the greatest of all trans-Atlantic physicians, studied stereoscopic views of people walking on the streets of Paris and New York to understand human locomotion as a model for limb prostheses [1]. A British physician, Richard L. Maddox, invented the first highquality dry photographic plate process in 1871 . The wet collodion plates previously in use required up to $10 \mathrm{~s}$ of stable (tripod) exposure and immediate processing. Collodio-bromide plates, marketed first in 1867, although somewhat 'dry', required $30 \mathrm{~s}$ exposures and had significant loss of sensitivity [2]. Maddox replaced collodion with a dry gelatine emulsion, prepared on glass, which he described in a letter to the British Journal of Photography in 1871. The editorial prelude labelled these emulsions 'the driest of the dry' and Maddox called them 'the result of somewhat careless experiments tried at first on an exceedingly dull afternoon.' [3]. He had abandoned wet collodion plates because he could not stand the smell of the ether necessary in their preparation. Maddox lamented that the pressures of clinical practice prevented further photographic experimentation. Although poor lighting required exposures of 30-90 s for his first dry plates, the Maddox method was reproducible, sensitive and quickly extended the range of photography to handheld cameras with exposures as short as $1 / 25$ of a second.

One of the first surgeons to substantively merge photography and surgery was A.G. Gerster (1848-1923), a Hungarian who arrived in the USA at 25 years of age to became one of the first and most successful full-time surgeons in New York City [4]. Gerster, doubly innovative, was an early amateur photographer who used his photographic skills to illustrate his landmark surgical textbook, The Rules of Aseptic and Antiseptic Surgery, which was first published in 1887 , only one year before George Eastman introduced the Kodak box camera. The
Fig. 1. The first illustration in Gerster's textbook [5]; Gerster is seen preparing a patient for mastectomy using aseptic technique.

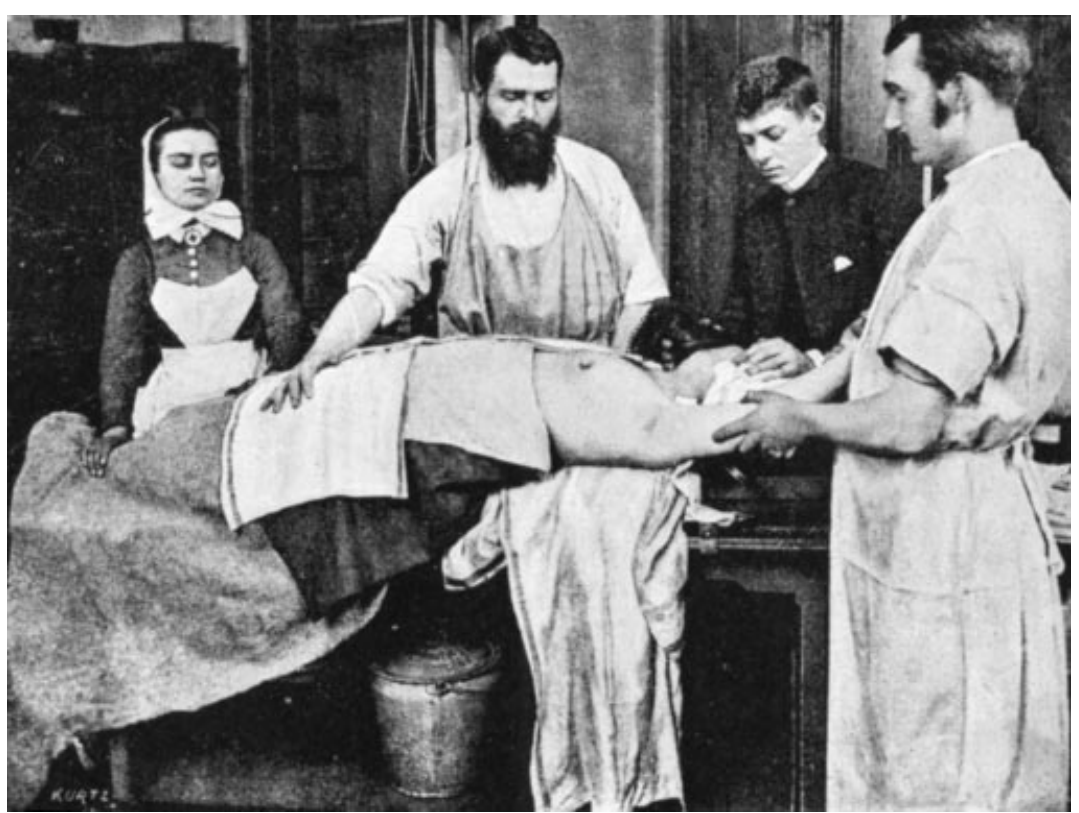

Accepted for publication 12 May 1998 

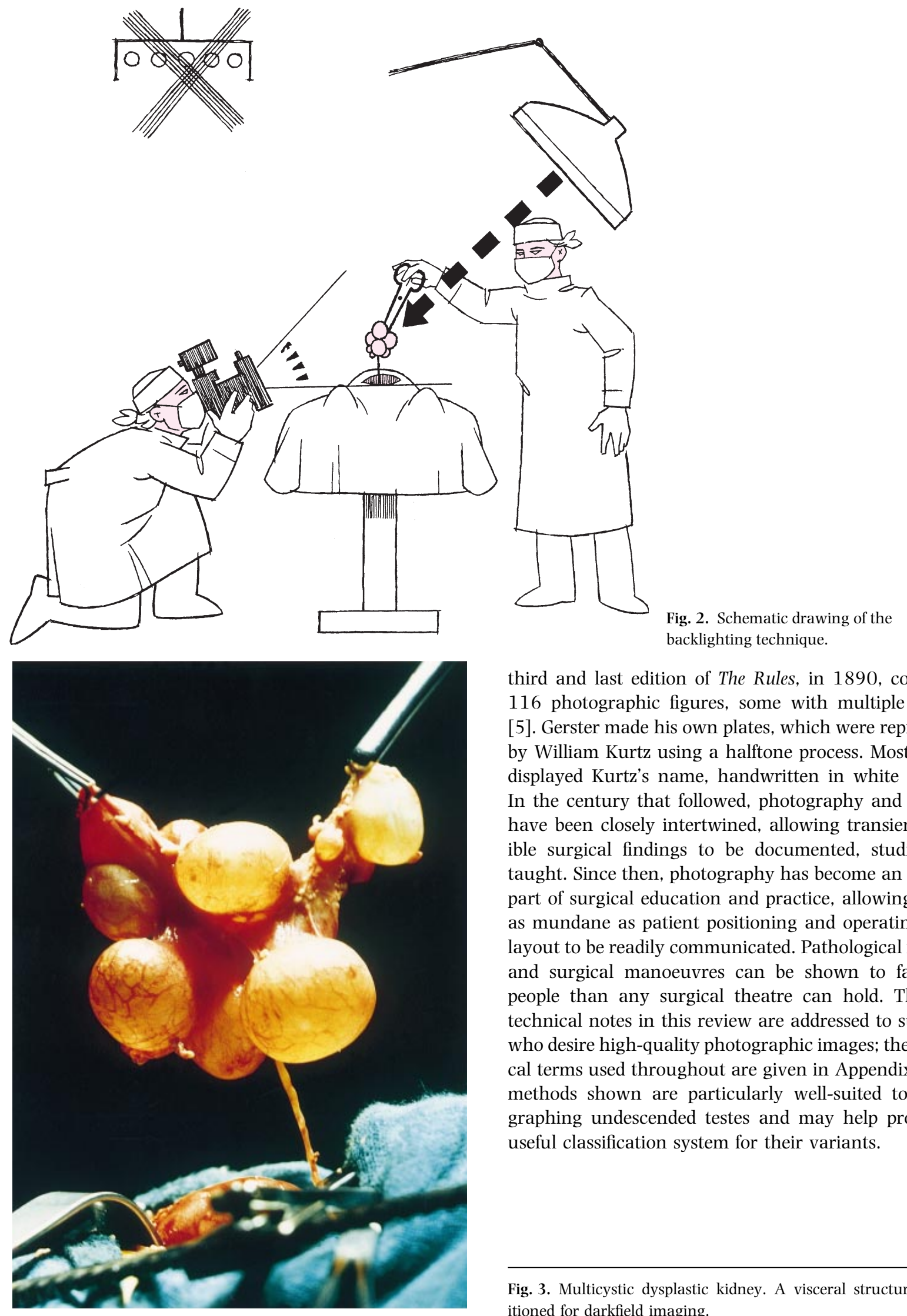

third and last edition of The Rules, in 1890, contained 116 photographic figures, some with multiple images [5]. Gerster made his own plates, which were reproduced by William Kurtz using a halftone process. Most figures displayed Kurtz's name, handwritten in white (Fig. 1). In the century that followed, photography and surgery have been closely intertwined, allowing transiently visible surgical findings to be documented, studied and taught. Since then, photography has become an integral part of surgical education and practice, allowing details as mundane as patient positioning and operating room layout to be readily communicated. Pathological findings and surgical manoeuvres can be shown to far more people than any surgical theatre can hold. The brief technical notes in this review are addressed to surgeons who desire high-quality photographic images; the technical terms used throughout are given in Appendix 1. The methods shown are particularly well-suited to photographing undescended testes and may help produce a useful classification system for their variants.

Fig. 3. Multicystic dysplastic kidney. A visceral structure is positioned for darkfield imaging.

(C) 1998 British Journal of Urology 82, 325-330 


\section{Methods}

We use $35 \mathrm{~mm}$ multiprogram single-lens reflex cameras with computerized shutter and aperture-priority, combined with a $100 \mathrm{~mm}$ macrolens $(1: 1)$, ring flash and 100 ASA colour-slide film. Other film types and film speeds may be useful for occasional situations, but we think it best to become adept with only one or two film types for most surgical photographic situations.

To isolate a subject with a dark background, the following method is used without a flash. The operating room overhead spotlight, positioned as shown Fig. 2, serves as a back-light or sidelight. When possible, the subject is lifted from the plane of the skin incision and turned toward the camera, orthogonally orientated to the film plane, thereby reducing blurred areas. Light orientation may vary, depending on the desired effect, from back-lighting to illuminating the upper edge of the subject. The other operating room lights should be dimmed to achieve a black background (Fig. 3). An aperture-priority program allows the depth of field to be increased or reduced, thereby isolating the main subject from the background. Setting a larger aperture reduces the depth of field, whereas a smaller aperture maximizes the depth of field in focus. The technique of lifting the subject away from the surgical field achieves detail of mesenteric and cystic structures and is particularly effective in the study of epididymal alterations in undescended testes.

In other situations, it may be neither desirable nor feasible to isolate the subject, and electronic flash illumination is useful. Photographing a deeply recessed structure calls for a ring flash and careful focus, accounting for the attendant depth of field. Here, an aperture-priority program may offer the best control over the depth of field. Some programmed cameras have a slow-shutter option with electronic flash that also increases the depth of field by permitting a smaller aperture for the given exposure.

Superficial structures call for careful framing of the anatomical object. Unnecessary blood on dirty towels and drapes detracts from the contrast, the intended subject and the overall aesthetics. A few extra moments to clean the field and define the subject are well spent. Various skin tones, e.g. when photographing hypospadias repairs, can have a surprising influence on image
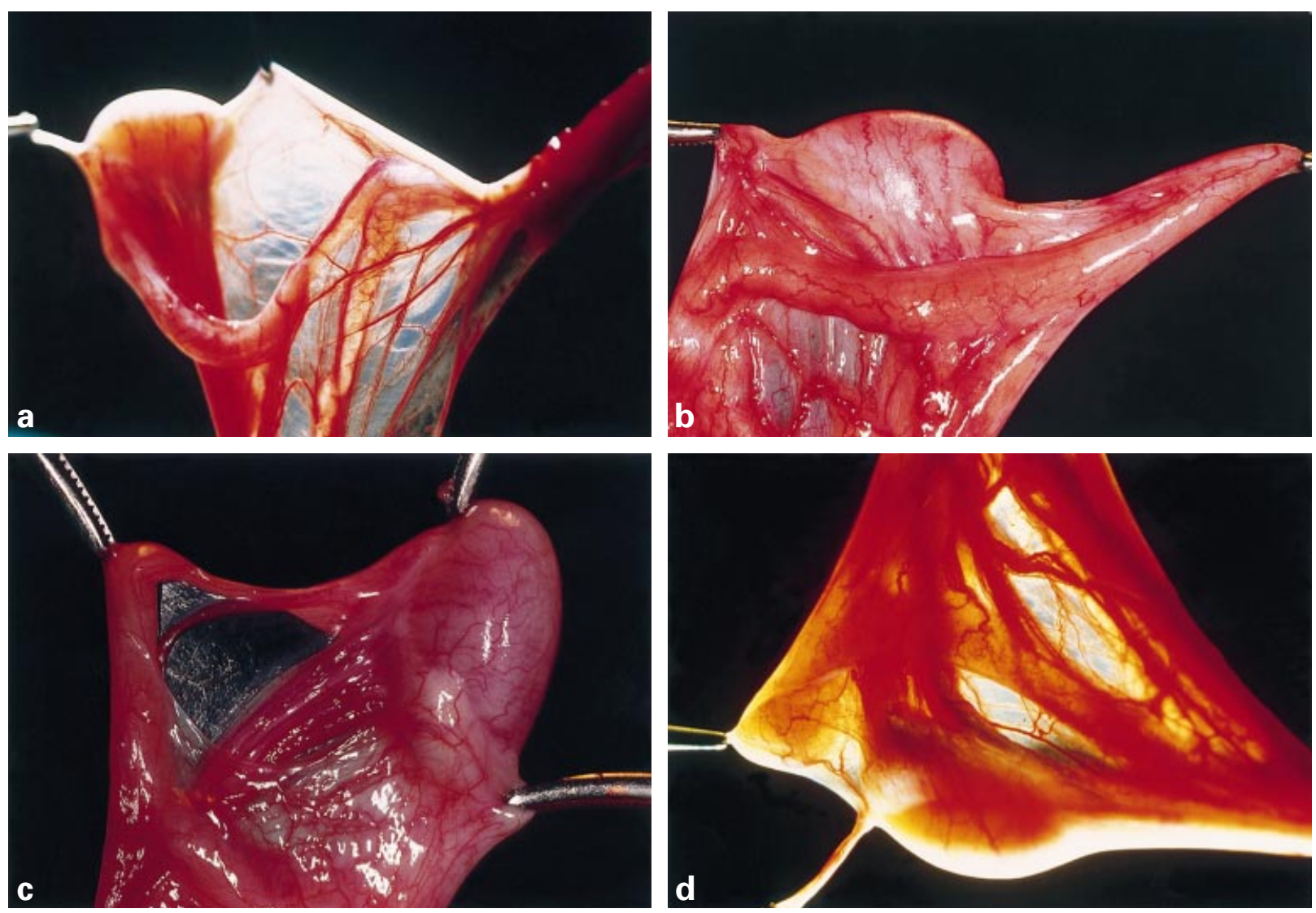

Fig. 4. Epididymal-vasal configurations in undescended testes: (a) nonfusion of the lower pole of the epididymis to the testis with a widely distracted epididymal body; (b) a long lower epididymal-vasal loop; (c) nonfusion of the upper pole of the epididymis to the testis (no rete testis); (d) completely distracted epididymis. 


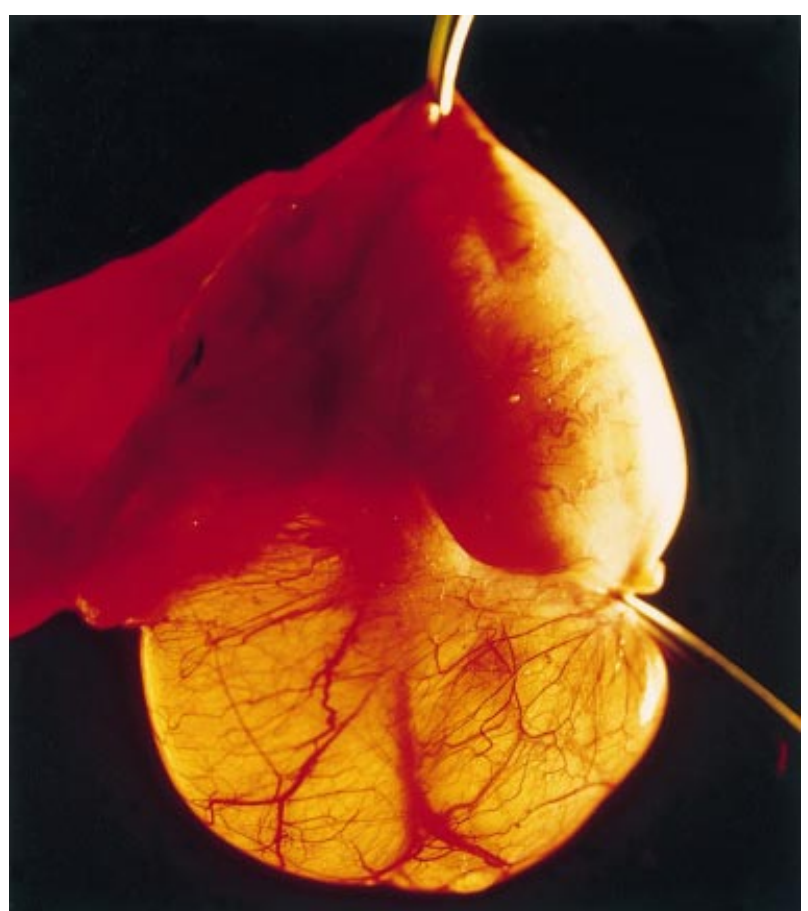

Fig. 5. Epididymal cysts.

quality. For example, with light skin situations a +1 or $2 \mathrm{EV}$ may be necessary for proper contrast, whereas a -1 or $2 \mathrm{EV}$ may be critical for darker complexions. Experimentation and bracketing will help the surgical photographer master the individual circumstances of anatomical structures, skin tones and equipment. With new equipment or new anatomical situations, it is best to take a series of shots even with programmed, aperturepriority or shutter-priority systems. This is carried out using the exposure-compensation setting and taking a first picture at zero, a second picture with $+1-2 \mathrm{EV}$ and a third with $-1-2 \mathrm{EV}$ settings. Once the optimal modes and parameters have been identified for each anatomical situation, the nurses in the operating room can take the photographs. The value of 'bracketing' the exposure in this way cannot be overemphasized, particularly for unique surgical situations or anatomical circumstances, where there may be no 'second chance'.

\section{Discussion}

The digital revolution in photography will have a tremendous impact on surgical photography; indeed the video camera has already broadened the influence of imaging in the operating room. Nonetheless, still photography will retain an important place in the imaging armamentarium. The modern multiprogram autoexposure electronic camera, with dedicated autoflash, obviates many technical problems for surgical photographers.
However, each anatomical situation produces individual conditions that are not perfectly imaged by a single programmed setting, therefore skilful intra-operative photography requires that the surgeon be equally adept with manual and programmed camera settings.

Intra-operative photographs allow anatomical variants to be captured for later classification and study. This has been useful in patients with undescended testes where the multiple pathological epididymal-vasal configurations have defied a widely used model (Figs 4 and 5). In nearly 150 years of still photography, the camera has become an indispensable part of surgical practice and education. New imaging technologies challenge older methods and provide improved tools for the operating theatre and the quieter moments of continuing education.

\section{Acknowledgements}

The authors are grateful to Mr Gianni Yacoumatos for Fig. 2.

\section{References}

1 Holmes OW. The human wheel, its spokes and felloes. Atlantic Monthly 1863; XI: 567-80

2 Newhall B. The History of Photography. 5th edn New York, NY: Museum of Modern Art 1997: 123-4

3 Maddox RL. An experiment with gelatino-bromide. $\mathrm{Br}$ J Photography 1871; 18: 422-3

4 Rutkow IM. The History of Surgery in the United States. San Francisco: Norman Publishing, 1988: 98-100

5 Gerster AG. The Rules of Aseptic and Antiseptic Surgery. New York: D. Appleton \& Co., 1890

\section{Authors}

F. Ferro.

D.A. Bloom, MD, Professor of Surgery, Chief of Pediatric Urology. Correspondence: Professor D.A. Bloom, 1500 East Medical Center Drive, University of Michigan, Ann Arbor, Michigan 48109-0330, USA.

\section{Appendix 1}

\section{A surgical photographic glossary}

Aperture is the size of the opening at the lens that allows light reflected from the subject to reach the film, and is designated as 'f stops'. Controlling the aperture is one way to control the exposure of the film, the other way being to control the duration of access of light to the film. The typical 'f stops' are $\mathrm{f} / 1.2, \mathrm{f} / 2.8, \mathrm{f} / 3.5, \mathrm{f} / 4, \mathrm{f} / 5.6$, $\mathrm{f} / 8, \mathrm{f} / 11, \mathrm{f} / 16$, and $\mathrm{f} / 22$. As these numbers increase, 
each step lets in half the light of its predecessor. The depth of field increases as the aperture decreases.

'Black-and-white' and colour film: When 'black and white' reproduction is intended for a book or journal, monochrome film is always best at the outset. Contrast is lost when a colour image is converted to black and white. Imaging in black and white requires particular attention to contrast, which the lighting and positioning should attempt to maximize. Conversely, colour is usually preferred for projection and illustration. Black and white films tend to be preferentially sensitive to blue; panchromatic films, sensitive to all visible light, are used for printing from colour negatives.

Colour temperature. The colour of light varies over the visible portion of the electromagnetic spectrum, which lies at 400-700 nm. Visible white light, travelling through a prism, breaks up into its colour components as the long waves (red) bend the least. The light source usually determines the dominant colours that make up the 'white light', although most of these hues are not discriminated by the human eye. Ordinary incandescent light bulbs produce a reddish light and fluorescent light is blue-green. Even daylight (sunlight) colour varies, tending to be blue in the morning, white at midday and pink near sunset. A scale expressing colour quality, expressed in Kelvins (K) or mireds (micro-reciprocal degrees) allows convenient and precise calibration of light sources that generally are perceived as white light to the naked eye. The Kelvin scale runs from yellow-red at several hundred Kelvins, to the blue hue of noonday sunlight at $\approx 10000 \mathrm{~K}$. The average daylight is $5500 \mathrm{~K}$ and it is this colour temperature to which daylight-type film and electronic flash systems are calibrated. Tungsten and halogen lamps have a colour balance of $3200 \mathrm{~K}$. Specific filters and films can reduce the 'unnatural' effect of the lighting source on the subject. Most operating theatre lights produce a yellowish effect when illuminating human tissues, unless electronic fill-in flash is used. Indirect reflection and transillumination of the backlighting method minimizes the lower colour temperature effect of the incandescent light without flash.

Contrast. permits clarity and definition of the image. This is particularly important in black and white photography and reproduction in journals and books. White, being the most reflective, gives the greatest response at the metering cell in the camera, more so than green and far more than black. If all the colours are arranged to form a so-called grey scale from white to black, the ratio between brightest and darkest would be 1:80. The logarithmic value of 80 is 1.9. Photographers found it convenient to subdivide the grey scale of 1.9 into six steps or exposure stops. These exposure stops can be aperture stops or shutter speeds, and their intervals along the logarithmic scale of 1.9 are 0.3 , if the extremes of black and white are disregarded. If all the colours in the average palate of a photograph (e.g. on a bright day in the summer) were mixed together an average grey would result, which would reflect $18 \%$ of the incident light. Exposure systems in most cameras are calibrated for this statistical average. However, if the subject has a higher reflectivity (e.g. the light-skinned patient with hypospadias) the metering system will assume $18 \%$ incident light reflection whereas it may be, e.g. $40 \%$ reflectivity, and the resulting photograph will be overexposed. The latitude of the film may not accommodate this gross underestimation. This can be prevented by using positive exposure compensation of 1-2 EV.

Correct exposure. The ideal combination of aperture and shutter speed exposes the film to the precise amount of reflected light such that the image captured perfectly mimics the image seen by the eye, or else creates the ideal image intended by the photographer.

Depth of field. is a distance interval from the lens within which all objects will be in reasonably sharp focus. As the distance of this interval increases, the depth of field increases. However, the major factor when photographing relatively near objects is the aperture; as this reduces (the f-stop increases) the depth of field also increases.

Digital imaging. The next generation of still photography. Light from the subject passes through a lens and falls on a charge-coupled device (CCD) which consists of multiple pixels that individually respond to the light they receive. The image is thus electronically mapped and stored on a disk in this digital form. The number of pixels available in CCDs to date is still far less than the number of silver halide grains in conventional still photography.

Exposure value (EV) This is an average luminance value or one exposure stop of 0.3 on the logarithmic scale. This step is equivalent to one shutter-speed interval or one aperture stop. Most film is flexible enough to permit some degree of imprecision or metering miscalculation. Typically, most negative print film tolerates five exposure stops, that can be broken down into $2 \mathrm{EV}$ of underexposure or $3 \mathrm{EV}$ of overexposure; slide film is less tolerant.

Flash vs available light. Available light in the operating room is generally artificial light, which tends to lend an unnatural appearance to skin tones. However, available light does offer shadows and the possibility of transillumi- 
nation, as evident in the cyptorchidism images (Fig. 4). Electronic flash provides uniform illumination and more natural skin tones. Shadows can still be created, offering a sense of depth, even with a ring flash, by using only the flash elements of a portion of the ring.

Focal length. is the distance from the lens (rear element) to the focal plane of the film. The practical importance of this is that the working distance of lens to subject is proportional to focal length. Therefore a long focallength lens, e.g. $105 \mathrm{~mm}$, allows the lens to be twice as far from the subject as a $50 \mathrm{~mm}$ lens. In addition, the longer focal length decreases the amount of background covered in the image and decreases the depth of field, permitting the subject to stand out more distinctly.

Focus. Focusing the lens creates a sharp image. Visual focus requires good optics and good eyes. Automatic focus can be incredibly precise, but the sensors can be fooled by monotonous contrasts and other situations. Traditionally, in very close or macrophotographic situations, manual focus has been preferred. In moving, respiring or peristaltic tissues, or in situations where the surgical photographer has a limited opportunity to focus in a deep or narrow recess, the automatic focus is quicker and more reliable than manual focus (especially for surgical photographers who wear bifocal glasses). With all of these constraints, plus a front-heavy macrolens with ring flash, the automatic focus feature is useful indeed.

F-stop. is the same as aperture. The f numbers are calculated by dividing the focal length of the lens by the aperture diameter. Thus, a $55 \mathrm{~mm}$ lens with an effective aperture of $5 \mathrm{~mm}$ has a relative aperture of $\mathrm{f} / 11$.
Print vs slide film. The correct technical terminology is negative vs transparency film. Print (negative) film is more forgiving in terms of exposure accuracy, in that printing can accommodate for some error. Slides (transparencies) require more accuracy, yet are more convenient in terms of economy, reviewing, projection and storage. However, it is better to err towards overexposure and retain detail otherwise lost.

Reproduction ratio is the magnification rate, i.e. the ratio between film-image size and subject size. For close-up surgical photography, reproduction ratios are generally fractional, e.g. $1: 2,1: 4$, or more. A reproduction ratio of $1: 1$ means that the subject is exactly the size as the image on the film. In the case of $35 \mathrm{~mm}$ film, this means that the subject can be no larger than $3.5 \mathrm{~cm}$. Macrophotography creates a magnified film image with a reproduction ratio in excess of $1: 1$, e.g. $1.2: 1,2: 1$, etc.

Shutter speed. determines the interval in which the film is exposed to the reflected light, and is one way to control the exposure value. Typical shutter speeds are $1 / 60$, $1 / 125,1 / 250$, and $1 / 500$ of a second and faster. Each step lets in half the light of its predecessor. Speeds slower than $1 / 30$ are generally accompanied by some camera shake. However, with synchronized electronic flash systems, the flash is far more rapid than the shutter speed. With some focal plane shutter systems the flash must be set at $1 / 30$ second or slower.

TTL Through the lens (TTL) metering systems provide the best co-ordination between aperture and shutter speed by measuring the exposure of light from the image that has passed through the lens. 